\title{
Regional Variation in Mammography Use among Insured Women 40-49 Years Old: Impact of a USPSTF Guideline Change
}

\author{
Jacqueline M. Hirth ${ }^{1}$, Yong-Fang Kuo ${ }^{2}$, Yu-Li Lin ${ }^{2}$ and Abbey B. Berenson ${ }^{1}$ \\ 1. Department of Obstetrics \& Gynecology, Center for Interdisciplinary Research in Women's Health, University of Texas Medical \\ Branch, Galveston 77555, TX, USA \\ 2. Preventive Medicine and Community Health, Office of Biostatistics, University of Texas Medical Branch, Galveston 77555, TX, USA
}

Received: April 62015 / Accepted: April 25, 2015 / Published: April 30, 2015.

\begin{abstract}
Purpose: The impact of the USPSTF (US Preventive Services Task Force) recommendation that 40-49 year old women should no longer routinely receive screening mammography in November 2009 in different regions of the US is unknown. Methods: The authors conducted a retrospective cohort study using medical claims from administrative health records from privately insured 40-59 year old women enrolled between 2005 and 2012 to evaluate biennial screening trends. Results: There was a slight decrease in mammography usage among 40-49 year old US women after the 2008-2009 biennial period $(p<0.001)$. There were some regional differences in mammography trends, with the West showing the greatest difference in odds of 40-49 year olds receiving a mammography in 2011-2012 compared to 2008-2009 (OR: 0.93; 95\% CI: 0.91-0.94). Although trends for 50-59 year olds mirrored that of 40-49 year olds, the younger age group had a stronger decline in 2009-2010 and 2010-2011. Conclusions: These findings show that USPSTF guideline changes made some differences in mammography usage among 40-49 year olds, but adherence was uneven across regions.
\end{abstract}

Key words: Mammography screening, screening guidelines adherence, cancer prevention.

\section{Introduction}

In November 2009, the USPSTF (US Preventive Services Task Force) updated breast cancer screening guidelines to recommend that women 40-49 years old should no longer routinely receive mammography for breast cancer screening [1]. These recommendations led to considerable controversy in both the medical field and the media [2,3]. Since the guidelines changed, some studies have found a decrease in mammography among 40-49 year olds, while others have observed no decrease [4-7]. In particular, a national retrospective analysis of privately insured women found that there was a modest decrease in monthly screening

Corresponding Author: Jacqueline M. Hirth, Ph.D., research fields: women's health, cancer prevention and population health. E-mail: jmhirth@utmb.edu. mammography rates, but that the rates then increased within a year after the recommendations were made [4]. However, data from the NHIS (National Health Interview Survey) showed that women reporting guideline compliant mammography use (were screened in the past 3 years) remained stable, even after the guidelines were changed [5]. Although there are already studies on this topic, there is little information about whether there are geographic differences in adherence to mammography screening trends, and whether differences could affect national observations.

Regional variation has been found to exist for adherence to cancer prevention practices. For example, access to mammography facilities has been found to vary between counties, with lower capacity available in those with populations that had lower education levels, increased poverty, and a higher proportion of women 
over 65 years of age [8]. Not only availability has an effect on utilization. Differences in demographics, cultural views, or legislation between regions may also have an effect on the utilization of preventive health practices on a population level. Compliance with guidelines for other preventive practices, such as human papillomavirus vaccination, varies by geography, with states in the South having particularly low rates [9]. Although it is currently not completely understood why these variations exist, state and regional policies, in particular, affect the uptake of preventive healthcare. It is important to determine whether regional variations in population screening behaviors exist because significant regional variations could be masked when conducting analyses on national-level data. The purpose of the present study is to examine whether there were regional variations in USPSTF mammography guideline adherence among 40-49 year old women.

\section{Materials and Methods}

This retrospective cohort study used health insurance claims from Clinformatics ${ }^{\mathrm{TM}}$ DataMart, a product of OptumInsight Life Sciences, Inc. (Eden Prairie, MN) administrative health data to select 40-59 year old women that were enrolled in a private insurance plan to examine the proportion who received mammography services during any biennial period of enrollment between 2005 and 2012. The dataset contains information on more than 45 million individuals, with $80 \%$ who purchase their health insurance through employers. The dataset has been de-identified, and contains no information about participants' socioeconomic status or ethnic background. The population in this dataset is roughly representative of the demographics of working US adults, but does have higher representation in the South. Data reflect health claims that have been paid by the insurance company, based on submissions from doctors across the United States.

Enrollees were selected to be included in the study if they were between the ages of 40 and 59 years of age, and had at least 2 years of enrollment with a 1 year look back period. Women with continuous coverage were eligible to be included at multiple time points if they were not diagnosed with breast cancer. Those who received a breast cancer diagnosis, confirmed through codes that indicated initiation of breast cancer therapy, were removed from the study after the date of diagnosis. Enrollees who participated in mammography screening were identified using 2013 HEDIS (Healthcare Effectiveness Data and Information Set), including: CPT codes 77055-77057, and 76090-76092; HCPCS codes G0202, G0204, and G0206; ICD-9-CM procedure codes 87.36-87.37; and UB revenue codes 0401 and 0403. HEDIS was used in order to gain the most comprehensive grouping of age-eligible women for the percent study. Although this also included some codes for diagnostic mammograms, it is possible that some providers may have used diagnostic codes instead of screening mammography codes. The authors excluded enrollees with a history of bilateral mastectomy and those who resided in Puerto Rico or other US territories from this study. More than 500,000 women in the 40-49 year age group were selected for each biennial period, and samples for the 50-59 year old cohort ranged from more than 426,000 in 2005-2006 to more than 512,000 in 2010-2011 (Table 1). The sampling using HEDIS reflects mammography rates that are about 3\% higher compared to sampling that removes cases with codes for a history of breast cancer in the past year and who had a record of a breast mass or cyst 30 days prior to the mammogram, or who had a diagnostic mammogram (Table 2). The present study was exempted from review by the University of Texas Medical Branch Institutional Review Board.

\subsection{Statistical Analyses}

For each biennial period between 2005 and 2012, the authors calculated the proportion of women from each age group (41-49 and 51-59 in the second year of each biennial period) who received a mammogram. 
Table 1 Cohort selection.

\begin{tabular}{|c|c|c|c|c|c|c|c|c|c|c|c|c|c|c|}
\hline & \multicolumn{7}{|c|}{ Aged $40-49$ years } & \multicolumn{7}{|c|}{ Aged 50-59 years } \\
\hline & $2005-2006$ & 2006-2007 & $2007-2008$ & $2008-2009$ & $2009-2010$ & $2010-2011$ & $2011-2012$ & $2005-2006$ & $2006-2007$ & $2007-2008$ & $2008-2009$ & $2009-2010$ & 2010-2011 & $2011-2012$ \\
\hline $\begin{array}{l}\text { 1. Aged } \\
40-49 / 50-59^{\ddagger} \\
\text { with complete } 2 \\
\text { years of } \\
\text { enrollment }\end{array}$ & 508,850 & 502,009 & 518,097 & 530,480 & 527,447 & 519,681 & 516,914 & 427,828 & 439,395 & 482,424 & 502,890 & 507,267 & 514,547 & 512,858 \\
\hline $\begin{array}{l}\qquad \downarrow \\
\text { 2. Did not have } \\
\text { a bilateral } \\
\text { mastectomy } \\
\text { before the end } \\
\text { of study period }\end{array}$ & 508,097 & 501,138 & 517,144 & 529,431 & 526,357 & 518,486 & 515,706 & 426,981 & 438,428 & 481,257 & 501,562 & 505,834 & 512,938 & 511,160 \\
\hline $\begin{array}{l}\text { 3. Exclude } \\
\text { those who } \\
\text { resided in } \\
\text { Puerto Rico or } \\
\text { US territories }\end{array}$ & 507,686 & 500,870 & 516,904 & 529,203 & 526,122 & 518,248 & 515,438 & 426,606 & 438,154 & 480,996 & 501,312 & 505,604 & 512,708 & 510,882 \\
\hline
\end{tabular}


Table 2 Codes used by HEDIS to identify breast cancer screening.

\begin{tabular}{ll}
\hline CPT & Description \\
\hline 77055 (historical code: 76090) & Mammography; unilateral \\
77056 (historical code: 76091) & Mammography; bilateral \\
77057 (historical code: 76092) & Screening mammography, bilateral \\
\hline HCPCS & \\
\hline G0202 & G0202-Screening mammography, producing direct digital image, bilateral, all views \\
G0204 & Diagnostic mammography, producing direct digital image, bilateral, all views \\
G0206 & Diagnostic mammography, producing direct digital image, unilateral, all views \\
\hline ICD-9-CM Procedure & \\
\hline 87.36 & Xerography of breast \\
87.37 & Other mammography \\
\hline UB Revenue & \\
\hline 0401 & Diagnostic mammography \\
0403 & Screening mammography \\
\hline
\end{tabular}

Cochran-Armitage trend tests were used to examine whether there were trends in mammography across time in the US and each of the four US Census regions (Midwest, Northeast, West, and South) within each age group examined. Region was controlled for in the final model for the US. Finally, because a woman could be selected in more than one biennial period, a GEE (generalized estimating equation) model was used to examine the odds of receiving a mammographic exam for each biennial period with autoregressive process order 1 as the covariance structure to account for clustering within individual cases. The biennial period 2008-2009 was the referent time frame, as that would be the biennial period that occurred before any change in population use of mammography could be expected to be observed. Analyses were stratified by age group so that differences across time between the age groups could be determined. All analyses were carried out using SAS Systems Inc. software version 9.3 (Cary, $\mathrm{NC)}$.

\section{Results}

The frequency of mammograms among 50-59 year olds remained consistent across time, with only slight decreases after 2008-2009 (Fig. 1a). A similar, but slightly stronger downward trend was noted among 40-49 year olds. After the 2008-2009 biennium, there were noticeable decreases in the proportion of 40-49 year old women who received a mammography in the Midwest (Fig. 1b), Northeast (Fig. 1c), and West (Fig. 1d), but not in the South (Fig. 1e). These trends were mirrored by those in the 50-59 year age group, but were not as strong among those women for whom routine screening recommendations are recommended biennially. There was a significant interaction between age group and time in the US as well as in each of the four US Census regions, which indicated that the effect of time on the odds of receiving a mammogram was different in the two age groups (analyses not shown).

Mammography rates ranged between $60 \%$ and $62 \%$ for privately insured $40-49$ year olds and $66 \%$ to over $67 \%$ among 50-59 year olds in each biennial period of enrollment (Table 3). Trend tests were significant for each region within the two age groups, with the exception of 40-49 year olds in the South.

Overall, compared to 2008-2009, 40-49 year olds in the US had small decreases in the odds of mammography in 2009-2010 and 2010-2011 before an increase occurred in 2011-2012, a pattern not shared with 50-59 year olds (Table 4). Some of the odds ratios that can be observed in Table 4 may appear to be slightly inconsistent with earlier figures. These differences are due to adjustment in the models for clustering effects within individual cases. The odds of 40-49 year old women receiving a mammogram were similar in the following biennial periods in the Northeast. There were 


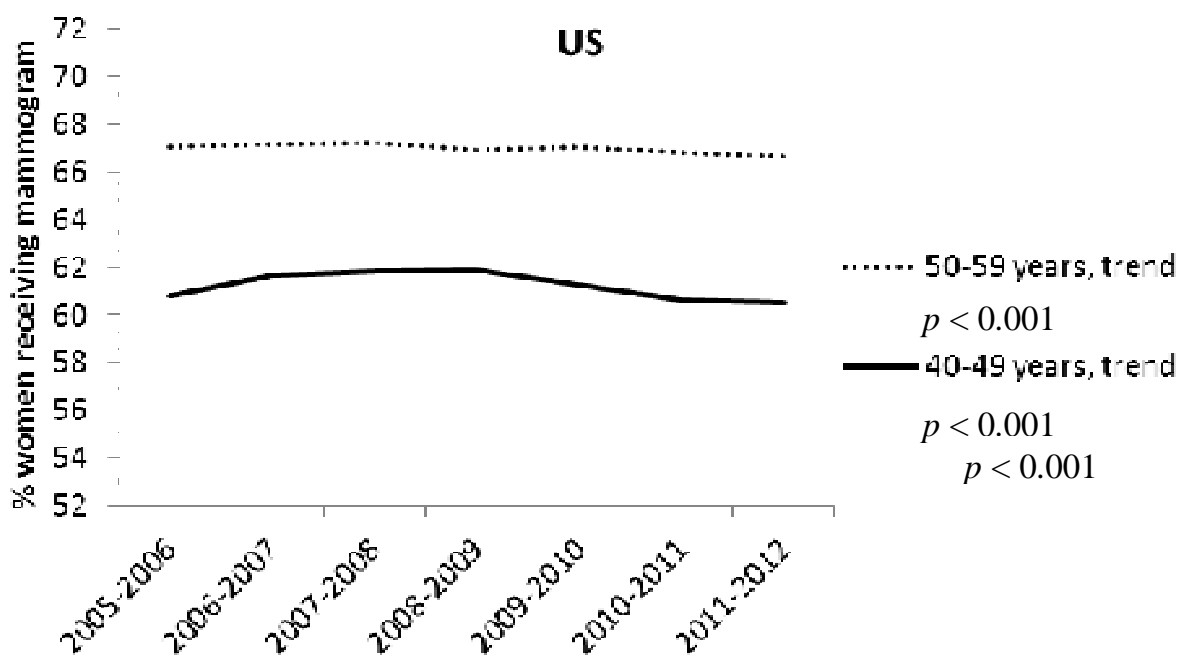

(a)

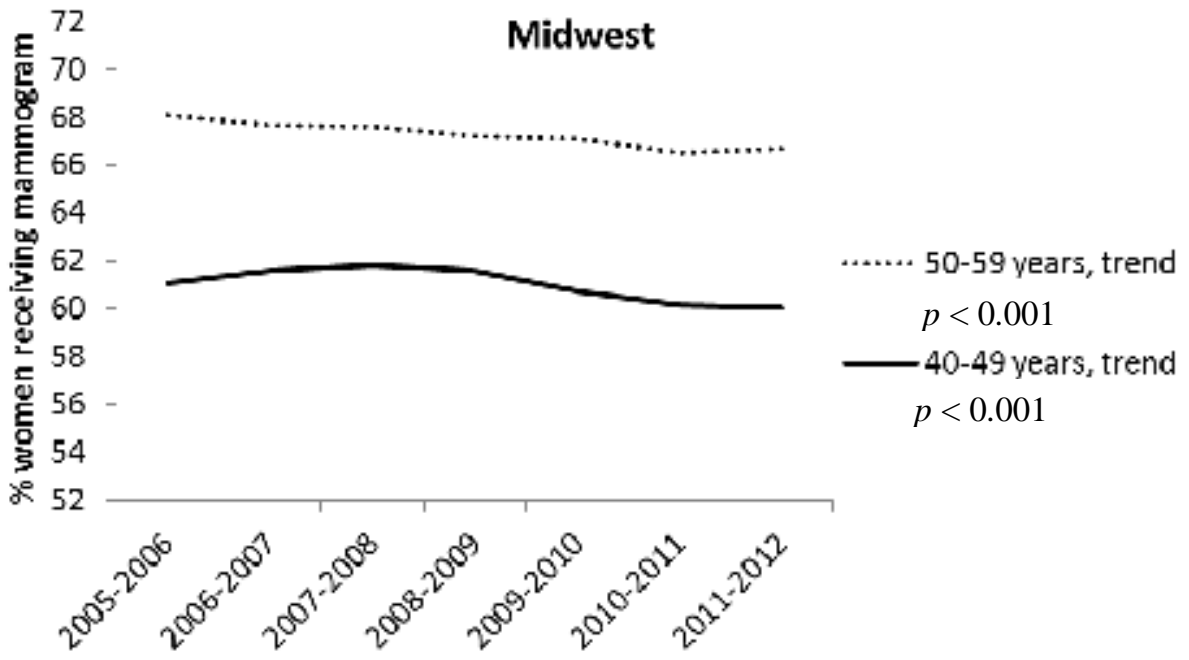

(b)

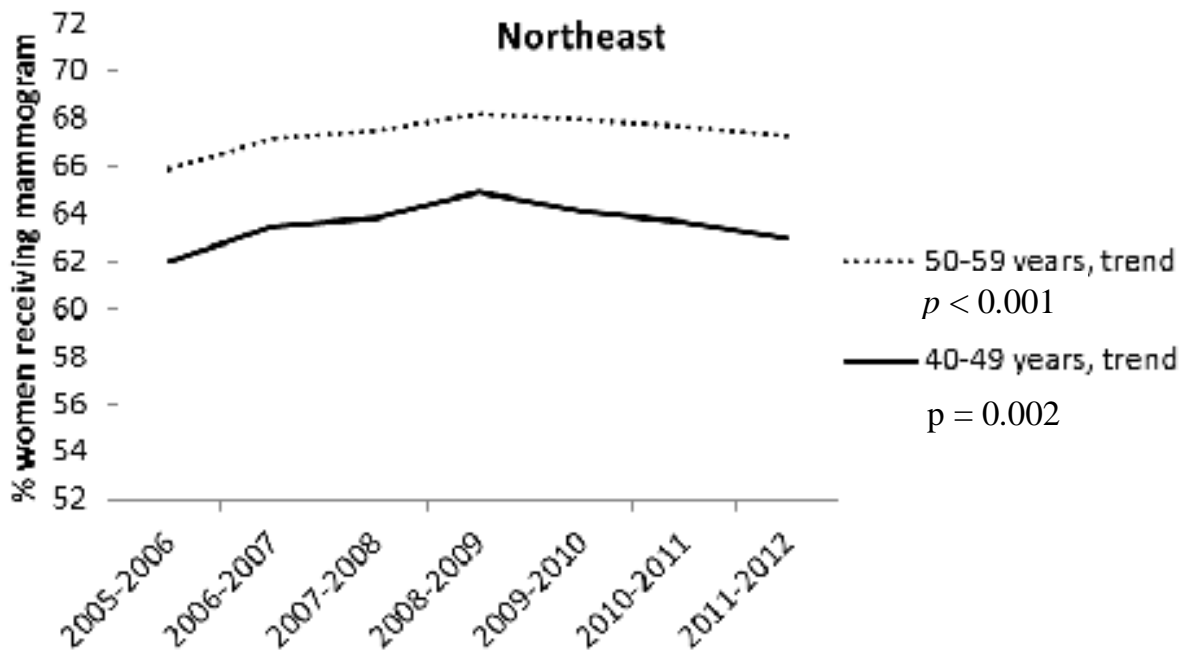

(c) 


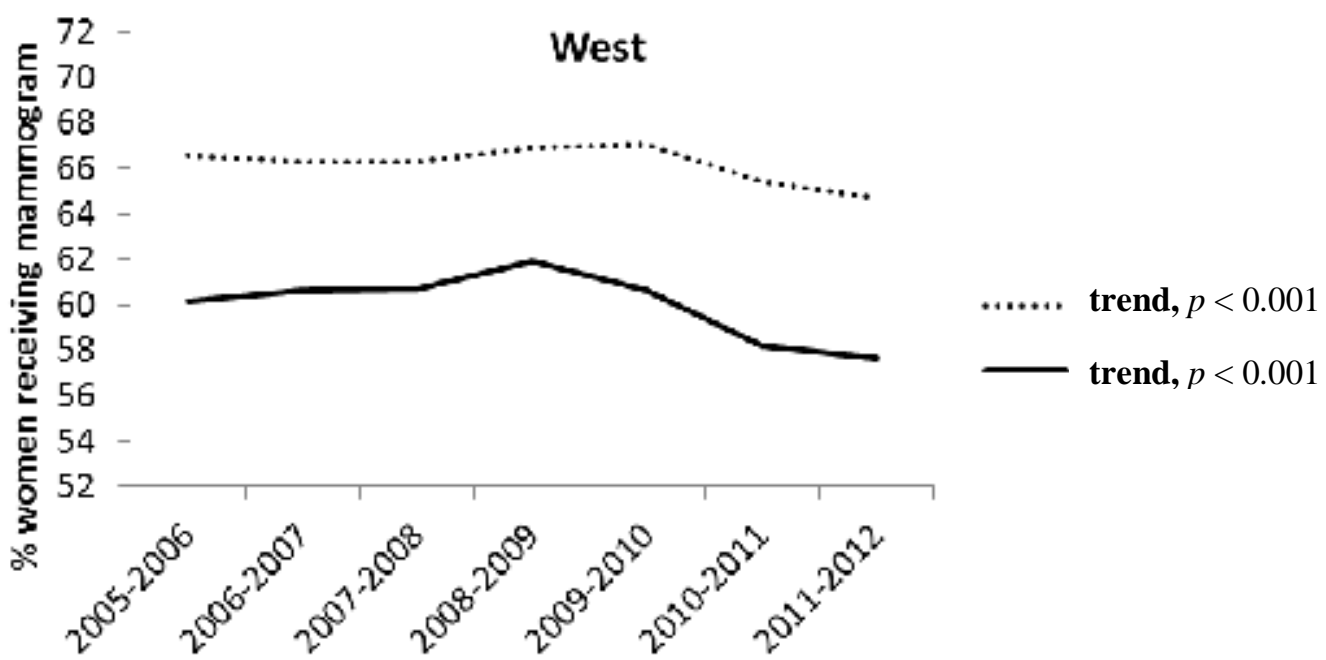

(d)

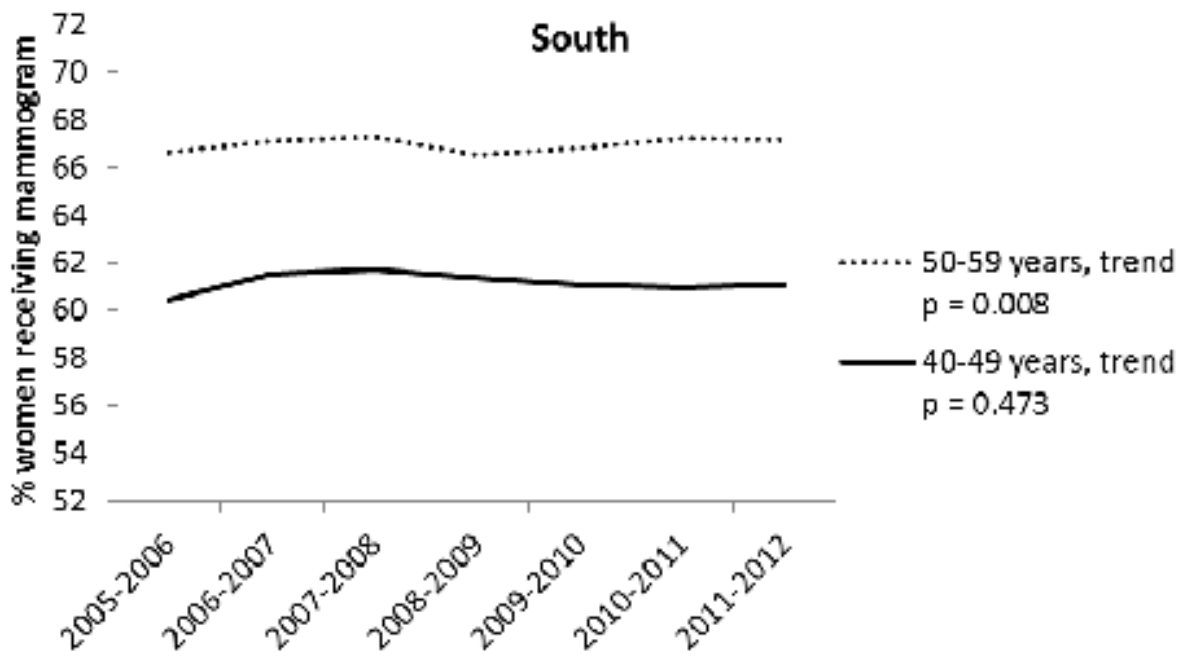

(e)

Fig. 1 Mammography rates in the US and 4 census regions, by year and age group.

modest decreases in the odds of 40-49 year old enrollees receiving a mammogram in 2009-2010 and 2010-2011 compared to 2008-2009 in the Midwest. The West showed decreased odds of receiving mammograms after 2008-2009 among 40-49 year olds. In general, the odds of receiving mammography remained similar across time among 50-59 year olds, with the exception of the West. There was a decline in the West among 50-59 year olds starting in 2010, but the magnitude is smaller than observed among 40-49 year olds.

\section{Discussion}

The national level results add an additional year of data (2012) to current reports in the literature and confirms that there was little change in mammography patterns among insured 40-49 year old females in the US, similar to what was observed in NHIS [5] and Behavioral Risk Factor Surveillance System data [10]. This information verifies that mammography usage has not dropped significantly as a longer amount of time has passed since the USPSTF issued the new mammography guidelines for 40-49 year olds. However, we did find that when data are aggregated across the US, changes in regional trends may be obscured.

Non-Western regions showed little to no decrease in the proportion of 40-49 year old women who received 
Table 3 Proportion of women who received mammography procedure by year, age group and region.

\begin{tabular}{|c|c|c|c|c|c|c|c|c|}
\hline & \multicolumn{7}{|c|}{$\%$ Women receiving mammogram } & \multirow{2}{*}{$p$, trend test } \\
\hline & 2005-2006 & 2006-2007 & 2007-2008 & 2008-2009 & 2009-2010 & 2010-2011 & 2011-2012 & \\
\hline \multicolumn{9}{|c|}{ Aged 40-49 } \\
\hline Overall & 60.78 & 61.65 & 61.83 & 61.89 & 61.26 & 60.65 & 60.49 & $<0.001$ \\
\hline Midwest & 61.10 & 61.61 & 61.84 & 61.59 & 60.72 & 60.17 & 60.04 & $<0.001$ \\
\hline Northeast & 62.02 & 63.50 & 63.86 & 64.92 & 64.10 & 63.67 & 62.99 & 0.002 \\
\hline South & 60.47 & 61.52 & 61.70 & 61.38 & 61.09 & 60.98 & 61.10 & 0.473 \\
\hline West & 60.19 & 60.65 & 60.69 & 61.89 & 60.63 & 58.22 & 57.67 & $<0.001$ \\
\hline \multicolumn{9}{|l|}{ Aged 50-59 } \\
\hline Overall & 67.04 & 67.15 & 67.24 & 66.93 & 67.03 & 66.81 & 66.66 & $<0.001$ \\
\hline Midwest & 68.08 & 67.65 & 67.61 & 67.23 & 67.13 & 66.56 & 66.69 & $<0.001$ \\
\hline Northeast & 65.92 & 67.15 & 67.50 & 68.18 & 67.98 & 67.68 & 67.29 & $<0.001$ \\
\hline South & 66.65 & 67.10 & 67.26 & 66.55 & 66.81 & 67.20 & 67.17 & 0.008 \\
\hline West & 66.61 & 66.33 & 66.32 & 66.93 & 67.05 & 65.42 & 64.69 & $<0.001$ \\
\hline
\end{tabular}

Table 4 Odds of having a mammogram within each biennial period, by region.

\begin{tabular}{lllllll}
\hline & \multicolumn{5}{c}{ Odds Ratio (95\% Confidence Interval) } \\
\cline { 2 - 7 } & $\mathrm{N}$ & US & Midwest & Northeast & South & West \\
\hline Aged 40-49 & & & & & \\
\hline $2005-2006$ & 507,686 & $0.95(0.94,0.95)$ & $0.96(0.95,0.97)$ & $0.89(0.87,0.91)$ & $0.95(0.94,0.96)$ & $0.93(0.92,0.95)$ \\
$2006-2007$ & 500,870 & $0.99(0.98,0.99)$ & $1.00(0.99,1.02)$ & $0.96(0.94,0.97)$ & $0.99(0.98,1.00)$ & $0.95(0.93,0.96)$ \\
$2007-2008$ & 516,904 & $0.99(0.98,0.99)$ & $1.00(0.99,1.01)$ & $0.97(0.96,0.98)$ & $0.99(0.99,1.00)$ & $0.96(0.95,0.97)$ \\
$2008-2009$ & 529,203 & 1.00 & 1.00 & 1.00 & 1.00 & 1.00 \\
$2009-2010$ & 526,122 & $0.98(0.97,0.98)$ & $0.97(0.96,0.98)$ & $0.98(0.97,0.99)$ & $0.99(0.99,1.00)$ & $0.95(0.93,0.96)$ \\
$2010-2011$ & 518,248 & $0.98(0.97,0.98)$ & $0.96(0.95,0.97)$ & $0.98(0.96,1.00)$ & $1.01(1.00,1.02)$ & $0.91(0.89,0.92)$ \\
$2011-2012$ & 515,438 & $1.01(1.01,1.02)$ & $0.99(0.98,1.00)$ & $1.00(0.98,1.02)$ & $1.06(1.05,1.06)$ & $0.93(0.91,0.94)$ \\
\hline Aged 50-59 & & & & & & \\
\hline $2005-2006$ & 426,606 & $1.02(1.01,1.03)$ & $1.05(1.04,1.06)$ & $0.94(0.92,0.96)$ & $1.02(1.01,1.03)$ & $1.01(1.00,1.03)$ \\
$2006-2007$ & 438,154 & $1.02(1.02,1.03)$ & $1.04(1.03,1.05)$ & $0.98(0.96,1.00)$ & $1.03(1.02,1.04)$ & $0.99(0.98,1.01)$ \\
$2007-2008$ & 480,996 & $1.01(1.00,1.01)$ & $1.01(1.00,1.02)$ & $0.98(0.97,1.00)$ & $1.01(1.01,1.02)$ & $0.98(0.97,1.00)$ \\
$2008-2009$ & 501,312 & 1.00 & 1.00 & 1.00 & 1.00 & 1.00 \\
$2009-2010$ & 505,604 & $1.01(1.00,1.01)$ & $1.01(1.00,1.02)$ & $1.00(0.99,1.02)$ & $1.02(1.01,1.02)$ & $1.00(0.98,1.01)$ \\
$2010-2011$ & 512,708 & $1.01(1.01,1.02)$ & $1.00(0.99,1.01)$ & $1.01(0.99,1.03)$ & $1.04(1.03,1.05)$ & $0.96(0.95,0.98)$ \\
$2011-2012$ & 510,882 & $1.03(1.02,1.03)$ & $1.01(1.00,1.03)$ & $1.00(0.98,1.02)$ & $1.06(1.05,1.07)$ & $0.96(0.94,0.97)$ \\
\hline
\end{tabular}

mammography screenings, whereas the West showed decreases and the South had a slight increase among both age groups. In Western states, it appeared that mammography was increasing among 40-49 year olds before the 2008-2009 biennium, then dropped off after that period of time, while changes were non-significant among 50-59 year olds until 2010-2011 when the odds of mammography decreased compared to the index biennium. The decreasing trends after the guideline changes in the West may be due to a greater adherence by providers in that region to USPSTF guidelines, as it has been found that guideline adherence varies among providers, especially when guideline conflicts occur [11]. However, it is important to note that changes in trends, although significant, were still very small. It is also important to note that changes in trends among 40-49 year olds were mirrored, in general, across time by $50-59$ year olds. This suggests that there may have been other factors affecting mammography usage, or that guideline changes may have also influenced mammography practices among women in the older age group. 
In addition to regional variations, it has been found that racial/ethnic variations exist in new mammography guideline compliance. Insured women of Asian descent had both annual and biennial decreases in mammography, while rates did not change among black women [12]. Together, these studies illustrate the importance of conducting analyses with the awareness that regional and other subgroup variations could be obscured by analyses that evaluates national trends.

One limitation of this study is that the results are not generalizable to all US women, as the sample was a cohort of continuously insured women. The authors were unable to evaluate demographics other than age and region of the US. Also, they did not exclude women whose mammography test was performed to evaluate symptoms.

The 2009 USPSTF recommendations had minimal impact on biennial mammography usage among 40-49 year old women in the US, but may have impacted mammography practices in the West, in particular. Follow up studies are needed to determine whether regional differences occurred among the uninsured, and whether changes result in missed cancer cases where mammography use may be declining among 40-49 year old women.

\section{Funding}

This work was supported by the Institute for Translational Sciences at the University of Texas Medical Branch, which is partially funded by a Clinical and Translational Science Award (UL1TR000071) from the National Center for Advancing Translational Sciences, National Institutes of Health. Dr. Hirth is a Scholar supported by a research career development award (K12HD052023: Building Interdisciplinary Research Careers in Women's Health Program-BIRCWH; Principal Investigator: Berenson) from the ORWH (Office of Research on Women's Health), the OD (Office of the Director), the NIAID (National Institute of Allergy and Infectious Diseases), the Eunice Kennedy Shriver NICHD (National
Institute of Child Health and Human Development) at the National Institutes of Health. The content is solely the responsibility of the authors and does not necessarily represent the official views of the National Institutes of Health.

\section{References}

[1] U.S. Preventive Services Task Force. 2009. "Screening for Breast Cancer: U.S. Preventive Services Task Force Recommendation Statement." Annals of Internal Medicine 2009 (151): 716-26.

[2] Allen, S. S., and Pruthi, S. 2011. "The Mammography Controversy: When should You Screen?" The Journal of Family Practice 60 (9): 524-31.

[3] Squiers, L. B., Holden, D. J., Dolina, S. E., Kim, A. E., Bann, C. M., and Ranaud, J. M. 2011. "The Public's Response to the U.S. Preventive Services Task Force's 2009 Recommendations on Mammography Screening." American Journal of Preventive Medicine 40 (5): 497-504.

[4] Wang, A. T., Fan, J., Van Houten, H. K., Tilburt, J. C., Stout, N. K., and Montori, V. M. 2014. "Impact of the 2009 US Preventive Services Task Force Guidelines on Screening Mammography Rates on Women in Their 40s." PLOS One 9 (3): e91399.

[5] Pace, L. E., He, Y., and Keating, N. L. 2013. "Trends in Mammography Screening Rates after Publication of the 2009 US Preventive Services Task Force Recommendations." Cancer 119 (14): 2518-23.

[6] Sprague, B. L., Bolton, K. C., Mace, J. L., Herschorn, S. D., James, T. A., and Vacek, P. M. 2014. "Registry-Based Study of Trends in Breast Cancer Screening Mammography before and after the 2009 US Preventive Services Task Force Recommendations.” Radiology 270 (2): 354-61.

[7] Howard, D. H., and Adams, E. K. 2012. "Mammography Rates after the 2009 US Preventive Services Task Force Breast Cancer Screening Recommendation." Preventive Medicine 55 (5): 485-7.

[8] Elkin, E. B., Atoria, C. L., Leoce, N., Bach, P. B., and Schrag, D. 2013. "Changes in the Availability of Screening Mammography, 2000-2010.” Cancer 119 (21): 3847-53.

[9] Rahman, M., McGrath, C. J., and Berenson, A. B. 2014. "Geographic Variation in Human Papillomavirus Vaccination Uptake among 13-17 Year Old Adolescent Girls in the United States." Vaccine 32 (21): 2394-8.

[10] Dehkordy, S. F., Hall, K. S., Roach, A. L., Rothman, E. D., Dalton, V. K., and Carlos, R. C. 2015. "Trends in Breast 
Cancer Screening: Impact of US Preventive Services Task

Force Recommendations." American Journal of Preventive Medicine Epub.

[11] Anderson, B. L., Pearlman, M., Griffin, J., and Schulkin, J. 2013. "Conflicting and Changing Breast Cancer Screening Recommendations: Survey Study of a National Sample of Ob-Gyns after the Release of the 2009 USPSTF
Guidelines." Journal for Healthcare Quality 35 (4): 25-35.

[12] Wharam, J. F., Landon, B., Zhang, F., Xu, X., Soumerai, S. and Ross-Degnan, D. 2015. "Mammography Rates 3 Years after the 2009 US Preventive Services Task Force Guidelines Changes.” Journal of Clinical Oncology 33 (9): 1067-74. 